ARTICLE

Received 20 Nov 2014 | Accepted 4 Aug 2015 | Published 10 Sep 2015

DOI: $10.1038 /$ ncomms9262 OPEN

\title{
Disparate ultrafast dynamics of itinerant and localized magnetic moments in gadolinium metal
}

\author{
B. Frietsch ${ }^{1,2}$, J. Bowlan ${ }^{1,2}$, R. Carley ${ }^{1,2}$, M. Teichmann ${ }^{1,2}$, S. Wienholdt ${ }^{3}$, D. Hinzke ${ }^{3}$, U. Nowak ${ }^{3}$, K. Carva ${ }^{4,5}$,
} P.M. Oppeneer ${ }^{4} \&$ M. Weinelt ${ }^{1}$

The Heisenberg-Dirac intra-atomic exchange coupling is responsible for the formation of the atomic spin moment and thus the strongest interaction in magnetism. Therefore, it is generally assumed that intra-atomic exchange leads to a quasi-instantaneous aligning process in the magnetic moment dynamics of spins in separate, on-site atomic orbitals. Following ultrashort optical excitation of gadolinium metal, we concurrently record in photoemission the $4 \mathrm{f}$ magnetic linear dichroism and $5 \mathrm{~d}$ exchange splitting. Their dynamics differ by one order of magnitude, with decay constants of 14 versus $0.8 \mathrm{ps}$, respectively. Spin dynamics simulations based on an orbital-resolved Heisenberg Hamiltonian combined with first-principles calculations explain the particular dynamics of $5 \mathrm{~d}$ and $4 \mathrm{f}$ spin moments well, and corroborate that the $5 \mathrm{~d}$ exchange splitting traces closely the $5 \mathrm{~d}$ spin-moment dynamics. Thus gadolinium shows disparate dynamics of the localized $4 \mathrm{f}$ and the itinerant $5 \mathrm{~d}$ spin moments, demonstrating a breakdown of their intra-atomic exchange alignment on a picosecond timescale.

\footnotetext{
${ }^{1}$ Fachbereich Physik, Freie Universität Berlin, Arnimallee 14, 14195 Berlin, Germany. ${ }^{2}$ Max-Born-Institut, Max-Born-Strasse 2a, 12489 Berlin, Germany.

${ }^{3}$ Fachbereich Physik, Universität Konstanz, 78457 Konstanz, Germany. ${ }^{4}$ Department of Physics and Astronomy, Uppsala University, PO Box 516,75120 Uppsala, Sweden. ${ }^{5}$ Charles University, Faculty of Mathematics and Physics, DCMP, Ke Karlovu 5, CZ-12116 Prague 2, Czech Republic. Correspondence and requests for materials should be addressed to M.W. (email: weinelt@physik.fu-berlin.de).
} 
U ltrafast spectroscopic techniques provide new insights into correlated materials by exciting specific subsystems and following their subsequent relaxation. For magnetic systems, this involves probing non-equilibrium spin and charge dynamics that cannot be reached through thermodynamic pathways. The magnetism in metals is determined by the interand intra-atomic exchange interactions ${ }^{1}$. The former couples the spin moments on neighbouring atoms and leads to the long-range order, while the latter enforces the formation of the atomic spin moment. Inter-atomic exchange is typically much weaker than intra-atomic exchange, which is the strongest and thus potentially fastest force in magnetism. These magnetic interactions can be scrutinized on the shortest timescales by employing femtosecond laser pulses. Such investigations have recently been reported for metallic ferromagnets, half-metallic ferromagnets, dilute magnetic semiconductors, as well as correlated magnetic oxides ${ }^{2-7}$. The response of multicomponent magnetic materials to a short, fs-laser pulse has been examined in an element-specific manner using time-resolved core-level magneto-optical techniques in the soft-X-ray and extreme ultraviolet (XUV) regime ${ }^{8-13}$. These investigations showed that the inter-atomic exchange interaction can be overcome on short timescales. Distinct magnetic responses were seen for the iron and nickel atoms in permalloy on a sub-picosecond timescale ${ }^{9}$. Probing $\mathrm{GdFeCo}^{8}, \mathrm{TbFe}^{14}, \mathrm{GdCo}$ $\mathrm{TbCo}^{13}$, and GdTb alloys ${ }^{15}$, different dynamics of the $\mathrm{Gd}$ (or $\mathrm{Tb}$ ) and $\mathrm{Fe}$ (or $\mathrm{Co}$ ) spins were observed. The experiments on multi-sublattice ferrimagnets thereby provide evidence for transient inter-atomic decoupling on the timescale of a few picoseconds. The much stronger intra-atomic exchange could thus far not be examined, as this requires a dedicated technique to selectively probe spin-polarized electrons in different orbitals on the same atom.

Gadolinium metal is an ideal system to offer deep insight in the operation of intra-atomic exchange under non-equilibrium conditions. The localized Gd 4 f electrons contribute most to the atomic moment with $7 \mu_{\mathrm{B}}$ per atom and spin-polarize the $5 \mathrm{~d}$ valence electrons, which contribute an additional $0.55 \mu_{\mathrm{B}}$, where $\mu_{\mathrm{B}}$ is the Bohr magneton. Because the $4 \mathrm{f}$ electrons of adjacent atoms have no spatial overlap, the neighbouring $4 \mathrm{f}$ moments align via intra-atomic (Hund's rule) exchange with the $5 \mathrm{~d}$ electrons, which-combined with the inter-atomic exchange between $5 \mathrm{~d}$ moments-leads to alignment of all moments (see Fig. 1a). The large intra-atomic exchange energy $J_{\text {int }}=130 \mathrm{meV}$, which corresponds to an exchange field of $\sim 4,000$ Tesla, is the background upon which quasi-instantaneous alignment $\left(\hbar / J_{\text {int }} \sim 6 \mathrm{fs}\right.$; $\hbar$ is the reduced Planck constant) of on-site moments and, hence, identical spin dynamics have been assumed thus far.

Time- and angle-resolved photoemission spectroscopy (ARPES) with ultraviolet light pulses has developed into a sensitive tool to probe details of the photoexcited state on the ultrafast timescale ${ }^{16-21}$. Here we apply time-resolved ARPES using higher-order harmonic radiation to study within a single experiment the two spin subsystems of Gd metal, which are coupled via intra-atomic exchange. We show within a single timeresolved photoemission experiment that $5 \mathrm{~d}$ exchange splitting and $4 \mathrm{f}$ magnetic linear dichroism (MLD) evolve upon 1.6-eV pulsed laser excitation on clearly different timescales with decay constants of 0.8 and 14 ps, respectively. We use first-principles calculations to derive the relation between the $5 \mathrm{~d}$ exchange splitting and the $5 \mathrm{~d}$ and $4 \mathrm{f}$ magnetic moments. The $a b$ initio calculated intra- and inter-atomic exchange constants enter an orbital-resolved Heisenberg Hamiltonian used to simulate the spin dynamics. With the respective coupling of $5 \mathrm{~d}$ and $4 \mathrm{f}$ spin systems to the electron and phonon heat baths (see Fig. 1a), the spin dynamics simulations explain the disparate dynamics of the $5 \mathrm{~d}$ and $4 \mathrm{f}$ magnetic moments very well, despite the large intra-atomic exchange coupling of $130 \mathrm{meV}$.

\section{Results}

Time-resolved photoemission spectroscopy. To scrutinize the intra-atomic exchange interaction on ultrashort timescales, we probed with orbital resolution the $4 \mathrm{f}$ and $5 \mathrm{~d}$ magnetization dynamics within a single photoemission experiment. We used an apparatus based on high-order harmonic generation ${ }^{22}$ to combine time-resolved ARPES with MLD in the angular distribution of photoelectrons ${ }^{23}$. The sample is a singlecrystalline 10 -nm-thick $\mathrm{Gd}(0001)$ film grown on a tungsten substrate. As shown in Fig. 1b, ARPES with a photon energy of $36.8 \mathrm{eV}$ gives access to the Tamm-like surface state, the transient exchange splitting of the $5 \mathrm{~d}$ minority and majority spin bands
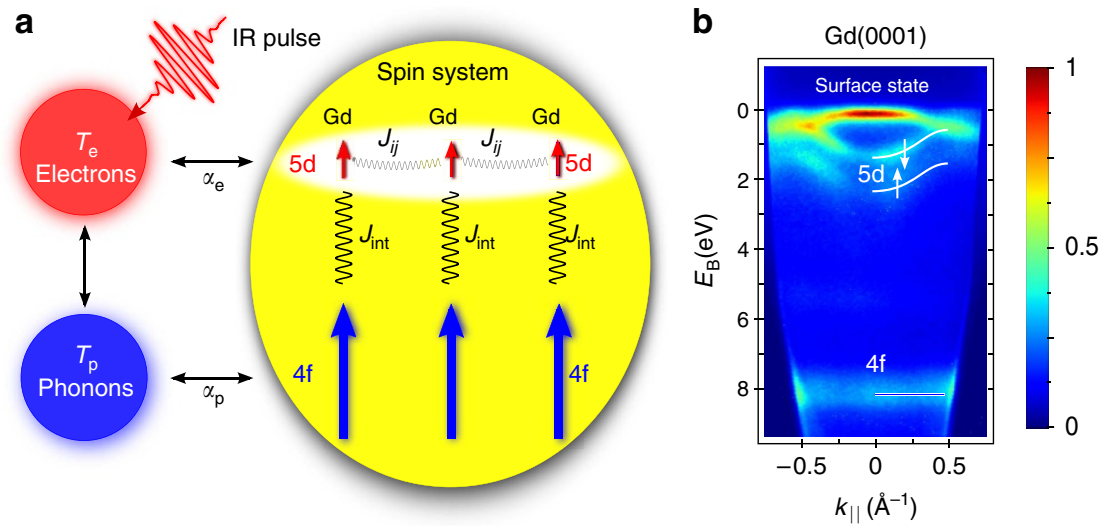

Figure 1 | Couplings of the gadolinium spin system. (a) The interaction of the different heat baths in an extended three-temperature model. After infrared laser excitation of the valence electrons, the whole system equilibrates by exchanging energy and momentum, indicated by the double arrows. The $5 \mathrm{~d}$ and $4 \mathrm{f}$ spin systems couple via inter- and intra-atomic exchange, where the intra-atomic exchange $J_{\text {int }}=130$ meV is much larger than the largest (nearest neighbour) inter-atomic exchange $J_{i j}=5.9 \mathrm{meV}$. In thermal equilibrium, the combination of inter-and intra-atomic exchange interactions mediates spin order in the $4 \mathrm{f}$ system via the delocalized $5 \mathrm{~d}$ valence bands. Upon femtosecond laser excitation, the dynamics of the $5 \mathrm{~d}$ spin system is dominated by the coupling $\alpha_{\mathrm{e}}$ to the hot valence electrons described by temperature $T_{\mathrm{e}}$, while the localized $4 \mathrm{f}$ spins couple only to the phonon bath at temperature $T_{\mathrm{p}}$ via $\alpha_{\mathrm{p}}$. (b) The binding energy versus parallel momentum map $E_{\mathrm{B}}\left(k_{\|}\right)$of $\mathrm{Gd}$ recorded with higher-order harmonic radiation (photon energy $36.8 \mathrm{eV}$ ) in time- and angle-resolved photoemission gives simultaneously access to the transient exchange splitting of the $5 \mathrm{~d}$ minority and majority spin bands ( $\downarrow$ and $\uparrow$, respectively) and the magnetic linear dichroism of the localized $4 \mathrm{f}$ state. Data are plotted on the displayed normalized false colour scale. 


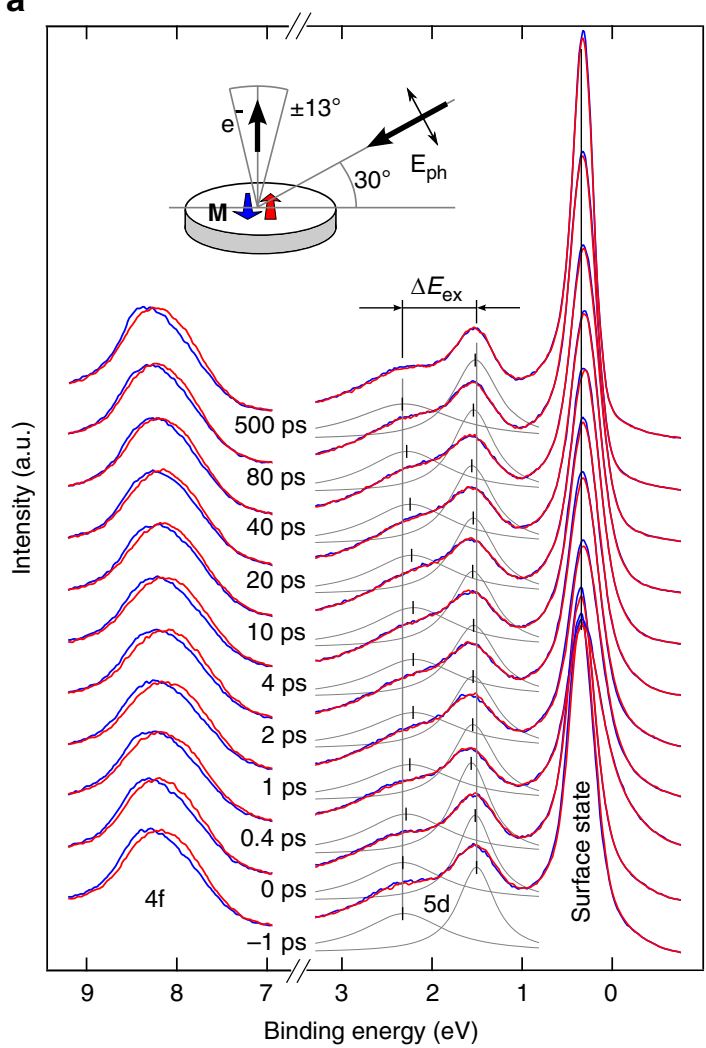

b

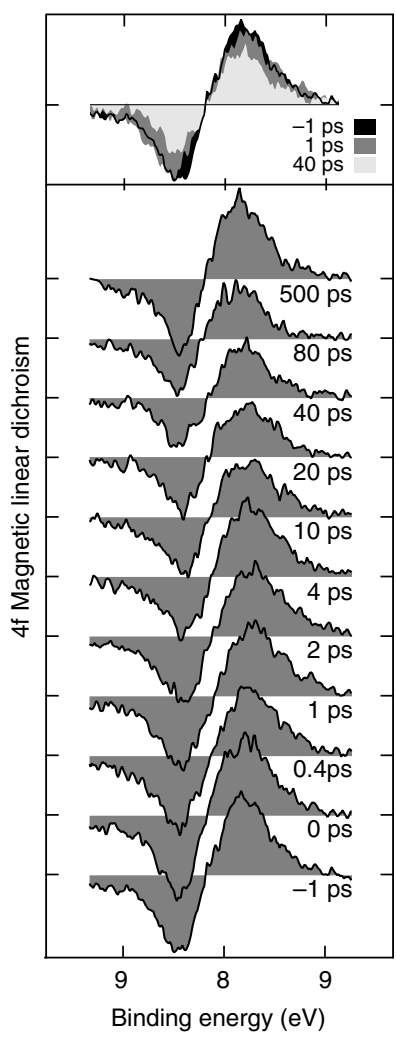

Figure 2 | Transient 5d exchange splitting and $\mathbf{4 f}$ linear magnetic dichroism of gadolinium. (a) The blue and red spectra show the Gd ARPES spectrum as a function of pump-probe delay recorded with p-polarized light in normal emission with a photon energy of $36.8 \mathrm{eV}$ for the two opposite in-plane magnetization directions. Clearly visible are the exchange-split (5d) valence bands at $2 \mathrm{eV}$ binding energy and the $4 \mathrm{f}$ core level at $8 \mathrm{eV}$, in addition to the surface state. The fitted majority and minority spin valence bands are indicated by grey peaks below the actual spectra, the peak positions are marked by black ticks. The inset depicts the experimental geometry for both magnetization directions $\mathbf{M}$ as indicated by the blue and red arrows. The angle of incidence of the pump and probe beams (bold black arrow) is $30^{\circ}$ off the surface plane and the electrons $\left(\mathrm{e}^{-}\right.$) are recorded in $\Gamma-\mathrm{M}$ direction $\pm 13^{\circ}$ with respect to the surface normal. The probe beam polarization, $E_{\mathrm{ph}}$, is indicated by the thin double-headed black arrow. (b) The magnetic linear dichroism (MLD) of the $4 \mathrm{f}$ electrons is obtained by integrating the absolute value of the difference of two spectra recorded for opposite in-plane magnetizations. Comparison of the signal at three different delays $(-1,1$ and $40 \mathrm{ps}$ as indicted by the colour scale) illustrates the slow, picosecond dynamics of the $4 \mathrm{f}$ magnetic moment.

( $\downarrow$ and $\uparrow$, respectively) and the localized $4 \mathrm{f}$ state. The Gd $5 \mathrm{~d}$ electrons and the surface state are excited by the $1.6-\mathrm{eV}$ laser pulse. This photon energy is too low to directly excite the occupied and unoccupied $4 \mathrm{f}$ states at $8 \mathrm{eV}$ and $-4 \mathrm{eV}$ binding energy $^{24}$. Figure $2 \mathrm{a}$ shows a series of photoemission spectra recorded for varying pump-probe delay extracted at the $\Gamma$ point (averaged over an acceptance window of $\pm 0.1 \AA^{-1}$ in Fig. 1b). As illustrated in the inset, we measured for each delay two photoemission spectra with opposite in-plane magnetization directions (red and blue lines) to determine the 4f MLD.

The occupied majority component of the Tamm-like surface state at binding energy $E_{\mathrm{B}} \leq 0.5 \mathrm{eV}$ indicates the high quality of the Gd film preparation. As the binding energy of the surface state at $\bar{\Gamma}$ is independent of the magnetization direction, it allows us to correct small space-charge shifts between individual measurements for opposite magnetization directions and equal delay. The small intensity change of the surface state for reversed magnetization is attributed to MLD. We observe a transient shift of the majority surface state towards the Fermi level by $\sim 50 \mathrm{meV}$, which is consistent with previous studies ${ }^{25,26}$.

The exchange-split minority and majority components of the $5 \mathrm{~d}$ valence band are seen at binding energies of 1.4 and $2.3 \mathrm{eV}$, respectively. With a probe photon energy of $36.8 \mathrm{eV}$, we measure the $\Delta_{2}$-like component in the 4 th Brillouin zone along the $\Gamma-\mathrm{M}$ direction. The valence band dispersion in Fig. $1 \mathrm{~b}$ agrees well with previous measurements at higher photon energies ${ }^{27}$ and the calculated bulk band structure ${ }^{28}$. The bulk character of these states was also confirmed by recent $a b$ initio calculations of a Gd $\operatorname{slab}^{29}$. Upon laser excitation, we observe a reduction of the exchange splitting (denoted $\Delta E_{\text {ex }}$ in Fig. 2a), which reaches its minimum within the first picosecond after laser excitation (see also Fig. 3). As substantiated below, the initial drop of the exchange splitting of the valence bands parallels the dynamics of the magnetic moment of the $5 \mathrm{~d}$ electrons.

To follow in addition the average $4 \mathrm{f}$ moment, we simultaneously recorded the intensity contrast of the $4 \mathrm{f}{ }^{7} \mathrm{~F}$ final-state spin-orbit multiplet at $\sim 8 \mathrm{eV}$ binding energy for opposite in-plane magnetization directions. The time evolution of the asymmetry is highlighted in Fig. 2b; the grey area is a measure of the transient $4 \mathrm{f}$ magnetic moment ${ }^{30}$. We note that photoemission probes the $5 \mathrm{~d}$ exchange splitting and $4 \mathrm{f} \mathrm{MLD}$ in the same sample volume defined by the inelastic mean free path of the photoelectrons of about three monolayers. Although photoemission is a surface-sensitive technique, the MLD contrast reflects mostly the $4 \mathrm{f}$ bulk magnetization, that is, the subsurface layers. High-resolution photoemission reveals a surface core-level shift of $0.3 \mathrm{eV}$ in the $\mathrm{Gd} 4 \mathrm{f}$ level with a bulk-to-surface intensity ratio of $3 / 2$ (refs 23,31). However, the atomic contribution to 


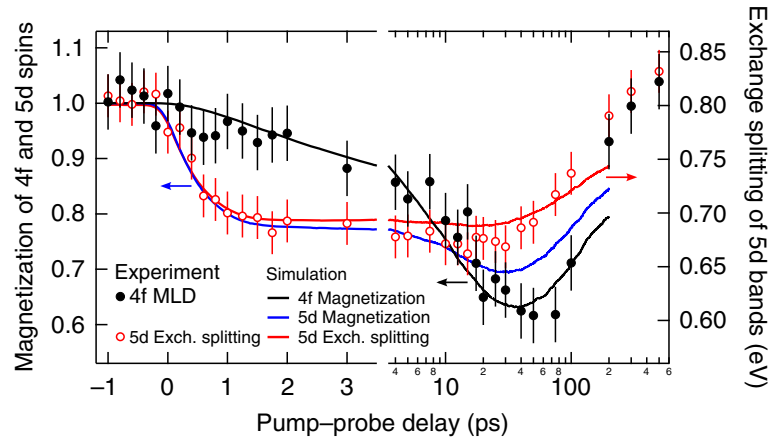

Figure 3 | Orbital-resolved spin dynamics in gadolinium. Measured exchange splitting of the $5 \mathrm{~d}$ bulk bands (red circles, right ordinate) and (normalized) magnetic linear dichroism of the $4 \mathrm{f}$ level (black circles, left ordinate) are shown as a function of pump-probe delay recorded with $100-f s$ XUV pulses. The position of the minority and majority spin valence band was extracted at $\pm 0.1 \AA^{-1}$ around $\Gamma$ using the fit procedure described in ref. 26. The MLD contrast is evaluated over the $4 f$ photoemission peak with an angular resolution of $0.5^{\circ}$ and integrated across the full detection range of $\pm 13^{\circ}$ to improve statistics. The error bars show two s.d. The error of the exchange splitting is obtained from the fits of the corresponding bands. The error of the MLD signal is given by the statistical noise of the spectral area at the $4 \mathrm{f}$ binding energy. Solid black and blue lines are the (normalized) $4 \mathrm{f}$ and $5 \mathrm{~d}$ magnetic moments calculated with our orbital-resolved spin Hamiltonian. The red solid line is the exchange splitting computed ab initio with the calculated $4 \mathrm{f}$ and $5 \mathrm{~d}$ magnetic moments of the spin dynamics simulations as input. Within the first few picoseconds after laser excitation, the dynamics of the exchange splitting and the $5 \mathrm{~d}$ orbital momentum are synchronous. The decoupling of the intra-atomic exchange is demonstrated by the significantly different demagnetization times of the $5 \mathrm{~d}$ and $4 \mathrm{f}$ spin system. Single exponential fits give time constants of 0.8 and 14 ps, respectively. Note that after $3.5 \mathrm{ps}$, the dynamics are displayed on a logarithmic scale to cover the cooling back to the initial sample temperature of $90 \mathrm{~K}$.

MLD due to the interference of the $\mathrm{d}$ and g photoemission final states is small at our photon energy since the $\mathrm{f}-\mathrm{g}$ dipole transition is $\sim 10$ times stronger than the $\mathrm{f}-\mathrm{d}$ counterpart ${ }^{31}$. Thus, the MLD signal mainly originates from photoelectron diffraction where the prevailing forward scattering only enhances the MLD bulk signal. This justifies comparison of the exchange splitting of the $5 \mathrm{~d}$ valence bands with the $4 \mathrm{f} \mathrm{MLD.} \mathrm{As} \mathrm{illustrated} \mathrm{in} \mathrm{Fig.} \mathrm{2b,} \mathrm{we}$ observe a clear reduction in MLD contrast at 40 ps delay, while it is only slightly changed at 1 ps delay (compared with the spectrum recorded before pumping at -1 ps delay).

The normalized exchange splitting and $4 \mathrm{f}$ MLD as a function of pump-probe delay are summarized in Fig. 3 by red and black circles, respectively. Their strikingly different temporal evolution reveals ultrafast decoupling of the intra-atomic exchange interaction. While the $5 \mathrm{~d}$ exchange splitting reaches its minimum after one picosecond, by which time the $5 \mathrm{~d}$ electron and phonon heat baths are nearly in equilibrium, the $4 \mathrm{f}$ magnetization continues to decrease until about 40 ps. The recovery of the magnetization occurs by the time the lattice and spins have comparable temperatures ${ }^{26}$. Fitting the initial collapse of the $5 \mathrm{~d}$ exchange splitting with a single exponential function yields a time constant of $\tau_{5 \mathrm{~d}}=0.8 \pm 0.1 \mathrm{ps}$. This agrees with the time constants from magneto-optical Kerr effect (MOKE) measurements $(0.85 \pm 0.05 \mathrm{ps})^{32}$ and our earlier work $(0.86 \pm 0.1 \mathrm{ps})^{26}$. For the $4 \mathrm{f}$ response, however, we find a much longer single exponential time constant of $\tau_{4 \mathrm{f}}=14 \pm 3 \mathrm{ps}$.

Orbital-resolved spin dynamics simulations. To shed light on the origin of the disparate magnetization dynamics of the $5 \mathrm{~d}$ exchange splitting and 4f spin system, we performed atomistic spin dynamics simulations. The $5 \mathrm{~d}$ moments and the corresponding exchange splitting are caused by the exchange field of the $4 \mathrm{f}$ electrons. Nonetheless, it has been shown experimentally ${ }^{33}$ and theoretically ${ }^{34}$ that their behaviour deviates significantly from a Stoner-like behaviour. This motivates us to treat the $4 \mathrm{f}$ and $5 \mathrm{~d}$ moments separately, leading to an orbital-resolved Heisenberg Hamiltonian including the intra-atomic interaction to go beyond the standard model with one fixed spin per atom. This approach was proposed recently and has been shown to describe very well the magnetization switching dynamics in ferrimagnets composed of transition-metal and rare-earth elements ${ }^{35}$; the predicted angular momentum transfer between the $3 \mathrm{~d}$ and $4 \mathrm{f}$ sublattices was confirmed by recent experiments ${ }^{13}$. Moreover, a simple three-temperature model proposed already for the first laserinduced demagnetization experiments on nickel ${ }^{36}$ has been shown to fail for the case of strong demagnetization ${ }^{37}$ or for experiments in $\mathrm{Gd}^{30}$, where the spin system itself is driven out of equilibrium $^{26}$. Consequently, Gd requires an orbital-resolved model to describe its two distinct spin systems, as illustrated in Fig. 1a. Here, the $5 \mathrm{~d}$ spins are coupled to the electronic temperature $\left(\alpha_{\mathrm{e}}\right)$ because the $5 \mathrm{~d}$ electrons are directly excited by the $1.6-\mathrm{eV}$ photons of the pump pulse. In contrast, the $4 \mathrm{f}$ electrons

are not perturbed by the pump pulse and thus couple, except through intra-atomic $4 \mathrm{f}-5 \mathrm{~d}$ exchange $J_{\text {int }}$, only to the phononic temperature of the system $\left(\alpha_{\mathrm{p}}\right)$.

Consequently, we construct the appropriate orbital-dependent spin Hamiltonian as

$$
\mathcal{H}=-\sum_{\langle i j\rangle} \frac{J_{i j}}{2} \mathbf{S}_{i} \cdot \mathbf{S}_{j}-\sum_{i} J_{\text {int }} \mathbf{S}_{i} \cdot \mathbf{S}_{i}^{\prime}-d_{z} \sum_{i}\left(S_{i}^{z}\right)^{2},
$$

where the $5 \mathrm{~d}$ and $4 \mathrm{f}$ spins are, in the classical limit, expressed by unit vectors $S_{i}$ and $\mathbf{S}_{i}^{\prime}$, representing the normalized $5 \mathrm{~d}$ and $4 \mathrm{f}$ magnetic moments, respectively. The first term describes the inter-atomic Heisenberg exchange between the $5 \mathrm{~d}$ spins at different sites $i, j$ of the hexagonal close-packed lattice. The second term accounts for the intra-atomic $5 \mathrm{~d}-4 \mathrm{f}$ exchange and the third term represents a uniaxial anisotropy. We consider Langevin dynamics, that is, we numerically solve the stochastic Landau-Lifshitz-Gilbert (LLG) equations of motion. For the $5 \mathrm{~d}$ spins, the LLG equation reads

$$
\dot{\mathbf{S}}_{i}=-\frac{\gamma}{\left(1+\alpha_{\mathrm{e}}^{2}\right) \mu_{\mathrm{s}}^{d}} \mathbf{S}_{i} \times \mathbf{H}_{i}(t)-\frac{\alpha_{\mathrm{e}} \gamma}{\left(1+\alpha_{\mathrm{e}}^{2}\right) \mu_{\mathrm{s}}^{d}} \mathbf{S}_{i} \times\left(\mathbf{S}_{i} \times \mathbf{H}_{i}(t)\right)
$$

where the phenomenological damping parameter $\alpha_{\mathrm{e}}$ describes the coupling between $5 \mathrm{~d}$ spins and the electronic heat bath, $\mu_{\mathrm{s}}^{d}$ is the $5 \mathrm{~d}$ spin moment and $\gamma$ denotes the gyromagnetic ratio. The effective field $\mathbf{H}_{i}(t)=\frac{\partial \mathcal{H}}{\partial \mathbf{S}_{i}}+\zeta_{i}(t)$ includes thermal fluctuations via the white-noise term $\zeta_{i}$ (ref. 38). The same equation describes the 4f spins $S^{\prime}$, however, with a coupling $\alpha_{\mathrm{p}}$ to the phononic heat bath. Separate values of $\alpha_{\mathrm{e}}$ and $\alpha_{\mathrm{p}}$ are not known in the literature, but it turns out that best agreement between simulation and experiment is achieved using different values, namely $\alpha_{\mathrm{e}}=0.00013$ and $\alpha_{\mathrm{p}}=0.0015$. Their average is in agreement with the Gilbert damping constant $\alpha=0.00044$ of Gd, known from ferromagnetic resonance ${ }^{39}$. The role of these values is analysed in detail in the Supplementary Discussion and Supplementary Fig. 1.

The exchange constants $J_{i j}$ and $J_{\text {int }}$ were calculated $a b$ initio using the density functional theory. To validate the exchange constants with our orbital-resolved spin model, we calculated the equilibrium net magnetization and the individual $5 \mathrm{~d}$ and $4 \mathrm{f}$ magnetizations versus temperature (Supplementary Fig. 2). 
Employing the exchange constants, we simulated a spin system with 45,696 atomic spins, taking into account exchange interactions up to the 22 nd nearest neighbour. We computed a Curie temperature $\left(T_{C}=299 \mathrm{~K}\right)$ close to the experimental value $\left(T_{\mathrm{C}}=293 \mathrm{~K}\right)$, implying that our orbital-dependent $a b$ initio exchange constants describe the localized and itinerant magnetism of Gd adequately. Finally, we mention that to compute the electron and phonon temperatures we employed a two-temperature model ${ }^{40}$ using material parameters similar to ref. 41 (see Supplementary Fig. 3 and Supplementary Table 1).

The results of the atomistic spin dynamics simulations for the $5 \mathrm{~d}$ and $4 \mathrm{f}$ moments are shown in Fig. 3 as blue and black solid lines, respectively. Our calculations clearly support a pronounced difference in the demagnetization times of the $4 \mathrm{f}$ spins with respect to the $5 \mathrm{~d}$ spins. To compare directly the exchange splitting predicted by our simulations with its experimental counterpart, we computed the average angle between the $4 \mathrm{f}$ and $5 \mathrm{~d}$ spin moments as a function of pump-probe delay. We then performed $a b$ initio calculations for this non-collinear arrangement of the two on-site moments, which gives us the electronic bands, and hence the value of the d-band exchange splitting. The relation between the average angle between the $4 \mathrm{f}$ and $5 \mathrm{~d}$ spin moments and the exchange splitting is given in Supplementary Fig. 4. Note that the $5 \mathrm{~d}$ exchange splitting computed ab initio closely follows the $5 \mathrm{~d}$ spin moment in the first $10 \mathrm{ps}$ (red and blue curves in Fig. 3, respectively) but deviates more when the $4 \mathrm{f}$ moments demagnetize more strongly. This permits us to draw conclusions on the time evolution of the $5 \mathrm{~d}$ spin moment from the exchange splitting. As can be seen from Fig. 3 the theoretical and measured $5 \mathrm{~d}$ exchange splitting are in good agreement, as are the theoretical and measured $4 \mathrm{f}$ demagnetization. The similarity of measured and simulated $5 \mathrm{~d}$ and $4 \mathrm{f}$ spin moments conclusively proves that despite the massive exchange field, the intra-atomic $5 \mathrm{~d}-4 \mathrm{f}$ exchange alignment is broken for tens of picoseconds.

\section{Discussion}

Comparing the measurements with the simulations described above provides the following understanding: laser excitation of the valence electrons in a single-crystalline Gd film creates nonequilibrium conditions between the $5 \mathrm{~d}$ and $4 \mathrm{f}$ spin systems, which persist for several tens of picoseconds. The vastly different energetic positions of the $5 \mathrm{~d}$ and $4 \mathrm{f}$ electrons in $\mathrm{Gd}$ are pivotal to the breakdown of the intra-atomic spin alignment on the ultrafast timescale. Initially, only the valence electronic system is heated rapidly by the pump pulse, leading to a fast loss of $5 \mathrm{~d}$ spin alignment, in spite of the huge exchange field exerted by the spinpolarized $4 \mathrm{f}$ electrons. The $4 \mathrm{f}$ spin system remains cold for much longer as it couples mainly to the phonon heat bath. Notably, as the $5 \mathrm{~d}$ electrons reach temperatures of a few thousand Kelvin (see Supplementary Fig. 3), their energy is sufficient to overcome the $4 \mathrm{f}$ exchange field. The transient breakdown of $5 \mathrm{~d}-4 \mathrm{f}$ intraatomic alignment only recovers on the slow, picosecond timescale of $4 \mathrm{f}$-spin-lattice relaxation ${ }^{42,43}$.

Performing spin dynamics simulations with various $\alpha_{p}$ and $\alpha_{e}$ damping parameters (see Supplementary Discussion) we find that increasing $\alpha_{\mathrm{p}}$ ten times does not strongly influence the initial $4 \mathrm{f}$ demagnetization, but affects the position of the $4 \mathrm{f}$ magnetization minimum at about $70 \mathrm{ps}$ (Fig. 3). Conversely, varying $\alpha_{\mathrm{e}}$ leads to a stronger change of the initial $5 \mathrm{~d}$ demagnetization, but does not influence the $5 \mathrm{~d}$ magnetization minimum at $40 \mathrm{ps}$. Thus, disparate spin dynamics of $4 \mathrm{f}$ and $5 \mathrm{~d}$ spins are consistently obtained here for a range of damping parameters. A different recent approach ${ }^{29}$ assumes that the $4 \mathrm{f}$ and $5 \mathrm{~d}$ moments in $\mathrm{Gd}$ cannot be treated separately and hence predicts the same demagnetization behaviour for $4 \mathrm{f}$ and $5 \mathrm{~d}$ moments, which, however, is not confirmed by our photoemission measurements.

The orbital-resolved spin-dynamics model also has its limitations. Phononic heat transport is neglected, which can cause a somewhat slower cooling in our simulations and thus a magnetization recovery on longer timescales ( $\geq 80 \mathrm{ps}$ ) than were measured. Note that the difference in the measured and computed recovery times of the $5 \mathrm{~d}$ and $4 \mathrm{f}$ spins is related to the slightly different equilibrium temperature dependence of the $5 \mathrm{~d}$ and $4 \mathrm{f}$ magnetizations (see Supplementary Fig. 2). As mentioned above, our spin-dynamics approach predicts angular momentum transfer between sublattices ${ }^{35}$. For the monoatomic Gd lattice, the transport of $5 \mathrm{~d}$ spin angular momentum occurs between atoms at differently excited depths of the sample via the inter-atomic exchange coupling. Our model does not include additional spin transport via laser-excited electrons, which we expect to be smaller for $\mathrm{Gd}$ than for the $3 \mathrm{~d}$ ferromagnets due to the smaller net magnetic moment of the Gd 5d valence electrons ${ }^{44}$.

The dynamics of the $4 \mathrm{f}$ MLD signal observed in ARPES is in contrast to a previous X-ray magnetic circular dichroism (XMCD) measurement at the $\mathrm{Gd} \mathrm{M}_{5}$-edge ${ }^{45}$, which probes the unoccupied $4 \mathrm{f}$ states. The latter experiment suggests that the demagnetization of the $4 \mathrm{f}$ system initially is as fast as that of the directly excited $5 \mathrm{~d}$ electrons measured with $\mathrm{MOKE}^{46}$. The XMCD experiment probes the whole film in transmission, and thus bulk properties. In addition, demagnetization will contain contributions from transport of optically excited electrons generated in the nonmagnetic Y-cap layers ${ }^{47}$ and the $\mathrm{Al}$ support into the polycrystalline Gd film. Such additional contributions are not present in our experiment.

Nonetheless, in the photoemission experiment we cannot rule out a small ultrafast drop of the MLD signal followed by a plateau between 0.2 and 2 ps. However, if the $5 \mathrm{~d}$ and $4 \mathrm{f}$ magnetic moments were in equilibrium, the normalized magnetization of the $5 \mathrm{~d}$ and $4 \mathrm{f}$ spin systems would lie on top of each other. Even within the error bars this is clearly not the case. Therefore, the data in Fig. 3 unambiguously support non-equilibrium between the two spin systems lasting for picoseconds. Our photoemission experiment probes the near-surface layers. The $5 \mathrm{~d}$ and $4 \mathrm{f}$ electrons have similar escape depth. In this sample volume, we demonstrate within one measurement disparate spin dynamics, despite the strong intra-atomic exchange. Unravelling the origin of the different $4 \mathrm{f}$ dynamics seen in photoemission and X-ray absorption asks for further experimental studies.

The electron and phonon subsystems equilibrate within $\sim 1.5 \mathrm{ps}$ (see Supplementary Fig. 3). Simultaneous to lattice heating, a strain field will evolve that propagates through the gadolinium film. The impact of such strain fields on the ultrafast magnetization dynamics has recently been discussed for nickel ${ }^{48}$. As crystalline gadolinium has a similar magnetostriction coefficient along its $c$ axis ${ }^{49}$, lattice strain may additionally contribute to the demagnetization dynamics. Note that the response of the polycrystalline film probed in XMCD can be quite different, since it is the average of a positive and negative magnetostriction parallel and perpendicular to the $c$ axis, respectively ${ }^{49}$. According to the density functional theory calculations ${ }^{25}$, the observed shift of the surface state to lower binding energies by $\sim 50 \mathrm{meV}$ (Fig. 2 and ref. 26) may point to an expansion of the surface interlayer spacing by about $20 \mathrm{pm}$. This value is similar to the lattice expansion observed when cooling down Gd bulk from $T_{\mathrm{C}}$ to about $100 \mathrm{~K}$ (refs 49,50). The anomalous lattice expansion of $\mathrm{Gd}$ is related to magnetostriction and indicates a repulsion between the ferromagnetic layers. Vice versa, expansion of the lattice upon laser excitation starting at the surface may stabilize the ferromagnetic state. These arguments 
are, however, challenged by the ultrafast ( $\leq 50 \mathrm{fs}$ ) and significant $(\geq 50 \%)$ drop of the surface-sensitive magnetic second harmonic signal $^{25}$, which indicates ultrafast demagnetization of the $\mathrm{Gd}$ surface layer ${ }^{41}$, as well as by the comparable dynamics of $5 \mathrm{~d}$ exchange splitting and $\mathrm{MOKE}^{32}$. These techniques probe nearsurface layers and bulk, respectively.

Our orbital-resolved spin dynamics simulations show that despite the strong intra-atomic exchange, disparate transient spin dynamics can occur. Recently, transient decouplings have been observed for the inter-atomic exchange in permalloy, which showed a $\sim 20$-fs shift in the transversal-MOKE response between the $\mathrm{Fe}$ and $\mathrm{Ni} \mathrm{M}$-edges ${ }^{9}$, as well as in GdFeCo alloy, where the inter-atomic exchange is much weaker $(\lesssim 3 \mathrm{meV})$ and the Gd-Fe decoupling lasted a few picosecond ${ }^{8}$. Here we show for the first time not only decoupling of the much stronger intra-atomic exchange $\left(J_{\text {int }}=130 \mathrm{meV}\right)$, but also that this decoupling lasts for about $40 \mathrm{ps}$. We note further that the intra-atomic exchange interaction has been considered previously in the context of laser-induced magnetization dynamics in ferromagnetic semiconductors ${ }^{51}$ and in laser-induced phase transitions in manganites ${ }^{7}$. In the former study, the influence of exchange coupling between localized and itinerant spin degrees of freedom was evaluated. In the latter study, quantum spin-flips mediated by the Hund's rule coupling of Mn $3 \mathrm{~d}$ states were proposed for fast switching of the magnetic order. In our approach, we extend in a different way beyond the classical spin limit of one spin per atom, by introducing exchange coupled, orbital-specific spins on an individual Gd atom. As confirmed by recent experiments ${ }^{13}$ and the presented simulations, our approach provides indeed a very good explanation of the orbitalselective spin dynamics. Since the large energy separation of the $5 \mathrm{~d}$ and $4 \mathrm{f}$ electrons is specific to $\mathrm{Gd}$, a similar observation of transient decoupling in other lanthanide metals will be difficult, as laser irradiation can rapidly heat both $4 \mathrm{f}$ and $5 \mathrm{~d}$ systems.

In conclusion, femtosecond laser-pulse excitation allows us to drive the $5 \mathrm{~d}$ and $4 \mathrm{f}$ spin systems of gadolinium metal out of equilibrium. Despite the strong intra-atomic exchange interaction, their demagnetization dynamics is characterized by time constants that differ by one order of magnitude. Their surprisingly disparate time evolution is well explained by orbital-resolved spin dynamics simulations based on exchange parameters calculated $a b$ initio. Our simultaneous examination of the localized and itinerant magnetism in Gd evidences that ultrafast laser stimulation of the valence electrons offers a route to transiently overcome the massive $4 \mathrm{f}-5 \mathrm{~d}$ exchange interaction. Understanding thereby the operation of fundamental magnetic interactions at ultrashort timescales and realizing the ability to manipulate them may have tremendous implications for future magnetic storage devices.

\section{Methods \\ Molecular beam epitaxy of Gd films on W(110). Single-crystalline, 10-nm-thick $\mathrm{Gd}(0001)$ films were grown epitaxially on a W(110) crystal at room temperature. The pressure during evaporation was $10^{-10}$ mbar. Subsequent annealing to $700 \mathrm{~K}$ allows the Gd lattice to relax and improves the film quality. Film thickness was calibrated by a quartz microbalance, film cleanliness and order were verified by low-energy electron diffraction and photoemission spectroscopy. The surface state intensity is a sensitive probe of the sample surface quality (see Fig. 2a and Supplementary Fig. 5).}

Time- and angle-resolved photoemission spectroscopy. Pump and probe pulses for the time-resolved ARPES experiment were derived from a femtosecond Ti:saphire chirped-pulse laser amplifier. The laser was running at $10 \mathrm{kHz}$, producing broadband pulses at a centre wavelength of $775 \mathrm{~nm}$. A beam splitter transmits $200-\mu \mathrm{J}$ pump pulses, which were incompletely compressed to $300 \mathrm{fs}$ duration in a separate compressor and adjusted in power and focus size. After compression to $45 \mathrm{fs}$, the remaining $1.3 \mathrm{~mJ}$ were focused into $100 \mathrm{mbar}$ argon to produce XUV probe pulses. A toroidal grating monochromator selected the 23rd harmonics with a photon energy of $36.8 \mathrm{eV}$. For the experiment we used p-polarized probe pulses with a duration of $100 \mathrm{fs}$ at a bandwidth of $150 \mathrm{meV}$. The pump pulse has a photon energy of $1.6 \mathrm{eV}$ and was stretched to $300 \mathrm{fs}$ pulse duration to reduce space-charge effects. The absorbed fluence is $3.5 \pm 1 \mathrm{~mJ} \mathrm{~cm}^{-2}$

The ARPES experiments were conducted at $3 \times 10^{-11} \mathrm{mbar}$ with a view-type $100-\mathrm{mm}$ hemispherical photoelectron analyzer. The exchange splitting of the $5 \mathrm{~d}$ bands was derived from energy distribution curves at normal emission (integrated at $\pm 0.1 \AA^{-1}$ in $\Gamma-M$ direction). The surface state, as well as minority and majority spin components of the $5 \mathrm{~d}$ band were fitted using Lorentzian line shapes (see Supplementary Fig. 5).

For the MLD signal, we corrected slight differences in the space-charge shift between the two magnetization directions by aligning the spectra at the surface state. This is appropriate because the surface state has vanishing Rashba splitting at the $\Gamma$-point ${ }^{31}$. The electron distribution curves for both magnetization directions were normalized in intensity before subtracting one from the other for each pumpprobe delay. The MLD signal is the integral over the absolute value of the intensity difference of the $4 \mathrm{f}$ state for opposite in-plane magnetization directions. In therma equilibrium this value is proportional to the $4 \mathrm{f}$ magnetization of the sample $\mathrm{e}^{30,52}$.

Density functional theory calculations. For the calculation of the intra- and inter-atomic exchange constants, we have adopted two different computational schemes. The intra-atomic exchange constant $J_{\text {int }}=130 \mathrm{meV}$ was calculated with the full-potential linear augmented plane wave method within the local spin density approximation (LSDA), employing the band-structure program ELK. Here, the $4 \mathrm{f}$ electrons were included in the valence states. This is necessary for describing correctly the interaction between the $5 \mathrm{~d}$ and $4 \mathrm{f}$ states. The program was modified to allow constraining the magnetizations of the spd and $4 \mathrm{f}$ states into an antiparallel alignment (see ref. 35 for details). The computed intra-atomic exchange constant is in good agreement with a previous calculation ${ }^{34}$.

For the $a b$ initio calculation of the inter-atomic exchange constants, we have found the approach in which the $4 \mathrm{f}$ electrons are treated as a part of the core states to be the most efficient. The self-consistent electronic structure was calculated using the tight-binding linear muffin-tin orbital method ${ }^{53}$, adopting the LSDA ${ }^{54}$ Treating the f electrons as localized core electrons notably helps to overcome some of the inaccuracies of the LSDA when applied to Gd, namely, its prediction of an antiferromagnetic ground state ${ }^{55}$ related to the positioning of the minority spin $4 \mathrm{f}$ states too close to the Fermi level ${ }^{28}$. Also, this approach has been successfully applied to predict the spontaneous volume magnetostriction in $\mathrm{Gd}^{34}$.

For the calculation of the inter-atomic exchange constants $J_{i j}(\leq 5.9 \mathrm{meV})$, we have employed the mapping of the magnetic behaviour of the real material onto an effective Heisenberg Hamiltonian ${ }^{56,57}$. Specifically, we have used the magnetic force theorem approach ${ }^{56}$, which allows infinitesimal changes of the total energy to be expressed in terms of the one-particle eigenvalues containing the non-selfconsistent changes of the effective one-electron potential accompanying the infinitesimal rotations of the spin quantization axes, that is, without any additional self-consistent calculations besides that for the collinear ground state. The resulting pair-exchange constants are given by

$$
\begin{aligned}
J_{i j}= & \frac{1}{\pi} \operatorname{Im} \int_{-\infty}^{E_{\mathrm{F}}} \mathrm{d} E \int_{\Omega_{i}} \mathrm{~d} \mathbf{r} \int_{\Omega_{i}} \mathrm{~d} \mathbf{r}^{\prime} B_{\mathrm{xc}}(\mathbf{r}) G^{\uparrow}\left(\mathbf{r}, \mathbf{r}^{\prime}, E^{+}\right) \\
& \times B_{\mathrm{xc}}\left(\mathbf{r}^{\prime}\right) G^{\downarrow}\left(\mathbf{r}^{\prime}, \mathbf{r}, E^{-}\right),
\end{aligned}
$$

where $E_{\mathrm{F}}$ denotes the Fermi level, $\Omega_{i}$ denotes the i-th atomic cell, $\sigma=\uparrow, \downarrow$ is the spin index, $E^{+}=\lim _{\alpha \rightarrow 0} E+\mathrm{i} \alpha, G^{\sigma}$ are spin-dependent one-electron retarded Green functions and $B_{\mathrm{xc}}$ is the magnetic field from the exchange-correlation potential. The validity of this approximation has been examined quantitatively in recent studies and it was found to be rather successful in explaining the thermodynamic properties of a broad class of magnetic materials ${ }^{58,59}$.

The exchange constants $J_{i j}$ have been computed up to the 29th nearestneighbour shell. We have used around a million $k$-points in the full Brillouin zone for energy points close to the Fermi level. In both tight-binding linear muffin-tin orbital and full-potential linear augmented plane wave calculations, the Gd lattice constant adopted was $3.629 \AA$ and the $c / a$ ratio, 1.597 .

Modelling of electronic and lattice temperature. To model the electronic and lattice temperature, we use the well-established two-temperature model ${ }^{40,60}$. Thereby perpendicular heat diffusion in the electronic sub-system is included. Furthermore, the energy flow into the spin system is taken into account by numerically calculating the time derivative of the Hamiltonian in equation (1) at every time step and adding it to the two-temperature model (see Supplementary Note).

\section{References}

1. Stöhr, J. \& Siegmann, H. C. Magnetism-From Fundamentals to Nanoscale Dynamics Vol. 152 (Springer Series in Solid State Science, 2006).

2. Müller, G. M. et al. Spin polarization in half-metals probed by femtosecond spin excitation. Nat. Mater. 8, 56-61 (2009). 
3. Koopmans, B. et al. Explaining the paradoxical diversity of ultrafast laser-induced demagnetization. Nat. Mater. 9, 259-261 (2010).

4. Boeglin, C. et al. Distinguishing the ultrafast dynamics of spin and orbital moments in solids. Nature 465, 458-461 (2010).

5. Wang, J. et al. Memory effects in photoinduced femtosecond magnetization rotation in ferromagnetic GaMnAs. Appl. Phys. Lett. 94, 021101 (2009).

6. Kirilyuk, A., Kimel, A. V. \& Rasing, T. Laser-induced magnetization dynamics and reversal in ferrimagnetic alloys. Rep. Prog. Phys. 76, 026501 (2013).

7. Li, T. et al. Femtosecond switching of magnetism via strongly correlated spin-charge quantum excitations. Nature 496, 69-73 (2013).

8. Radu, I. et al. Transient ferromagnetic-like state mediating ultrafast reversal of antiferromagnetically coupled spins. Nature 472, 205-208 (2011).

9. Mathias, S. et al. Probing the timescale of the exchange interaction in a ferromagnetic alloy. Proc. Natl Acad. Sci. USA 109, 4792-4797 (2012).

10. Vodungbo, B. et al. Laser-induced ultrafast demagnetization in the presence of a nanoscale magnetic domain network. Nat. Commun. 3, 999 (2012).

11. Pfau, B. et al. Ultrafast optical demagnetization manipulates nanoscale spin structure in domain walls. Nat. Commun. 3, 1100 (2012).

12. Graves, C. E. et al. Nanoscale spin reversal by non-local angular momentum transfer following ultrafast laser excitation in ferrimagnetic GdFeCo. Nat. Mater. 12, 293-297 (2013).

13. Bergeard, N. et al. Ultrafast angular momentum transfer in multisublattice ferrimagnets. Nat. Commun. 5, 3466 (2014).

14. Khorsand, A. R. et al. Element-specific probing of ultrafast spin dynamics in multisublattice magnets with visible light. Phys. Rev. Lett. 110, 107205 (2013).

15. Eschenlohr, A. et al. Role of spin-lattice coupling in the ultrafast demagnetization of $\mathrm{Gd}_{1-\mathrm{x}} \mathrm{Tb}_{\mathrm{x}}$ alloys. Phys. Rev. B 89, 214423 (2014).

16. Perfetti, L. et al. Time evolution of the electronic structure of $1 t-\mathrm{TaS}_{2}$ through the insulator-metal transition. Phys. Rev. Lett. 97, 067402 (2006).

17. Schmitt, F. et al. Transient electronic structure and melting of a charge density wave in $\mathrm{TbTe}_{3}$. Science 321, 1649-1652 (2008)

18. Rohwer, T. et al. Collapse of long-range charge order tracked by time-resolved photoemission at high momenta. Nature 471, 490 (2011).

19. Smallwood, C. L. et al. Tracking Cooper pairs in a cuprate superconductor by ultrafast angle-resolved photoemission. Science 336, 1137 (2012).

20. Zhang, W. et al. Ultrafast quenching of electron-boson interaction and superconducting gap in a cuprate superconductor. Nat. Commun. 5, 4959 (2014).

21. Wegkamp, D. et al. Instantaneous band gap collapse in photoexcited $\mathrm{VO}_{2}$ due to photocarrier doping. Phys. Rev. Lett. 113, 216401 (2014).

22. Frietsch, B. et al. A high-order harmonic generation apparatus for time- and angle-resolved photoelectron spectroscopy. Rev. Sci. Inst. 84, 075106 (2013).

23. Starke, K. Magnetic Dichroism in Core-Level Photoemission. Springer Tracts in Modern Physics (Springer, 2000).

24. Fauster, T. \& Himpsel, F. J. Energy dependence of cross sections in inverse photoemission. Phys. Rev. B 30, 1874-1876 (1984).

25. Loukakos, P. A. et al. Dynamics of the self-energy of the $\mathrm{Gd}(0001)$ surface state probed by femtosecond photoemission spectroscopy. Phys. Rev. Lett. 98, 097401 (2007).

26. Carley, R. et al. Femtosecond laser excitation drives ferromagnetic gadolinium out of magnetic equilibrium. Phys. Rev. Lett. 109, 0574012 (2012).

27. Döbrich, K. M., Bostwick, A., Rotenberg, E. \& Kaindl, G. Change of the Fermi surface of Gd metal upon magnetic ordering as seen via angle-resolved photoelectron spectroscopy. Phys. Rev. B 81, 012401 (2010).

28. Kurz, P., Bihlmayer, G. \& Blügel, S. Magnetism and electronic structure of hcp Gd and the Gd(0001) surface. J. Phys. Condens. Matter 14, 6353 (2002).

29. Sandratskii, L. M. Exchange splitting of surface and bulk electronic states in excited magnetic states of Gd: first-principles study. Phys. Rev. B 90, 184406 (2014).

30. Melnikov, A. et al. Nonequilibrium magnetization dynamics of gadolinium studied by magnetic linear dichroism in time-resolved $4 f$ core-level photoemission. Phys. Rev. Lett. 100, 107202 (2008).

31. Krupin, O. Dichroism and Rashba Effect at Magnetic Crystal Surfaces of Rare-Earth Metals. PhD. thesis, Freie Univ. Berlin (2004). http://www.diss.fu-berlin.de/diss/ receive/FUDISS_thesis_000000003802.

32. Sultan, M., Atxitia, U., Melnikov, A., Chubykalo-Fesenko, O. \& Bovensiepen, U. Electron- and phonon-mediated ultrafast magnetization dynamics of $\mathrm{Gd}(0001)$. Phys. Rev. B 85, 184407 (2012).

33. Maiti, K., Malagoli, M. C., Dallmeyer, A. \& Carbone, C. Finite temperature magnetism in Gd: evidence against a Stoner behavior. Phys. Rev. Lett. 88, 167205 (2002).

34. Khmelevskyi, S., Turek, I. \& Mohn, P. Spontaneous volume magnetostriction and non-Stoner behavior of the valence band in pure hcp Gd. Phys. Rev. B 70, 132401 (2004)

35. Wienholdt, S., Hinzke, D., Carva, K., Oppeneer, P. M. \& Nowak, U. Orbitalresolved spin model for thermal magnetization switching in rare-earth-based ferrimagnets. Phys. Rev. B 88, 020406 (2013).
36. Beaurepaire, E., Merle, J.-C., Daunois, A. \& Bigot, J.-Y. Ultrafast spin dynamics in ferromagnetic nickel. Phys. Rev. Lett. 76, 4250-4253 (1996).

37. Kazantseva, N., Nowak, U., Chantrell, R., Hohlfeld, J. \& Rebei, A. Slow recovery of the magnetisation after a sub-picosecond heat pulse. Europhys. Lett. 81, 27004 (2008).

38. Nowak, U. in Handbook of Magnetism and Advanced Magnetic Materials. (eds Kronmüller, H. \& Parkin, S.) Vol. 2 (John Wiley \& Sons, 2007).

39. Platow, W., Anisimov, A. N., Dunifer, G. L., Farle, M. \& Baberschke, K. Correlations between ferromagnetic-resonance linewidths and sample quality in the study of metallic ultrathin films. Phys. Rev. B 58, 5611-5621 (1998).

40. Anisimov, S. I., Kapeliovich, B. L. \& Perelman, T. L. Electron emission from metal surfaces exposed to ultrashort laser pulses. Sov. Phys. JETP 39, 375-377 (1974).

41. Bovensiepen, U. Coherent and incoherent excitations of the $\operatorname{Gd}(0001)$ surface on ultrafast timescales. J. Phys. Condens. Matter 19, 083201 (2007).

42. Vaterlaus, A., Beutler, T. \& Meier, F. Spin-lattice relaxation time of ferromagnetic gadolinium determined with time-resolved spin-polarized photoemission. Phys. Rev. Lett. 67, 3314-3317 (1991).

43. Hübner, W. \& Bennemann, K. H. Simple theory for spin-lattice relaxation in metallic rare-earth ferromagnets. Phys. Rev. B 53, 3422-3427 (1996).

44. Battiato, M., Carva, K. \& Oppeneer, P. M. Theory of laser-induced ultrafast superdiffusive spin transport in layered heterostructures. Phys. Rev. B 86, 024404 (2012).

45. Wietstruk, M. et al. Hot-electron-driven enhancement of spin-lattice coupling in $\mathrm{Gd}$ and $\mathrm{Tb} 4 f$ ferromagnets observed by femtosecond x-ray magnetic circular dichroism. Phys. Rev. Lett. 106, 127401 (2011).

46. Sultan, M., Melnikov, A. \& Bovensiepen, U. Ultrafast magnetization dynamics of Gd(0001): bulk versus surface. Phys. Status Solidi B 248, 2323 (2011).

47. Eschenlohr, A. et al. Ultrafast spin transport as key to femtosecond demagnetization. Nat. Mater. 12, 332-336 (2013).

48. Kim, J.-W., Vomir, M. \& Bigot, J.-Y. Ultrafast magnetoacoustics in nickel films. Phys. Rev. Lett. 109, 166601 (2012).

49. Bozorth, R. M. \& Wakiyama, T. Magnetostriction and anomalous thermal expansion of single crystals of gadolinium. J. Appl. Phys. 34, 1351-1352 (1963)

50. Darnell, F. J. Temperature dependence of lattice parameters for Gd, Dy and Ho. Phys. Rev. 130, 1825-1828 (1963).

51. Cywiński, L. \& Sham, L. J. Ultrafast demagnetization in the $s p$ - $d$ model: a theoretical study. Phys. Rev. B 76, 045205 (2007).

52. Beaulieu, N. et al. Probing ultrafast dynamics in electronic structure of epitaxial Gd(0001) on W(110). J. Electron Spectrosc. Relat. Phenom. 189, 40-45 (2013).

53. Turek, I., Drchal, V., Kudrnovský, J., Šob, M. \& Weinberger, P. Electronic Structure of Disordered Alloys, Surfaces and Interfaces (Kluwer, 1997).

54. von Barth, U. \& Hedin, L. A local exchange-correlation potential for the spin polarized case. J. Phys. C Solid State Phys. 5, 1629-1642 (1972)

55. Turek, I., Kudrnovský, J., Bihlmayer, G. \& Blügel, S. Ab initio theory of exchange interactions and the Curie temperature of bulk Gd. J. Phys. Condens. Matter 15, 2771-2782 (2003).

56. Liechtenstein, A. I., Katsnelson, M. I., Antropov, V. P. \& Gubanov, V. A. Local spin density functional approach to the theory of exchange interactions in ferromagnetic metals and alloys. J. Magn. Magn. Mater. 67, 65-74 (1987).

57. Halilov, S. V., Eschrig, H., Perlov, A. Y. \& Oppeneer, P. M. Adiabatic spin dynamics from spin-density-functional theory: application to $\mathrm{Fe}, \mathrm{Co}$ and $\mathrm{Ni}$. Phys. Rev. B 58, 293-302 (1998).

58. Turek, I., Kudrnovský, J., Drchal, V. \& Bruno, P. Exchange interactions, spin waves, and transition temperatures in itinerant magnets. Philos. Mag. 86, 1713-1752 (2006).

59. Kudrnovský, J., Drchal, V. \& Bruno, P. Magnetic properties of fcc Ni-based transition metal alloys. Phys. Rev. B 77, 224422 (2008).

60. Kaganov, M. I., Lifshitz, I. M. \& Tanatarov, L. V. Relaxation between electrons and the crystalline lattice. Sov. Phys. JETP 4, 173-178 (1957)

\section{Acknowledgements}

This project was supported by the Deutsche Forschungsgemeinschaft, the Leibniz Graduate School Dynamics in New Light, the Helmholtz Virtual Institute Dynamic Pathways in Multidimensional Landscapes and the European Commission via the Collaborative Project FEMTOSPIN (Grant No. 281043). We from Konstanz acknowledge the funding by the Center for Applied Photonics at the University of Konstanz, and those in Uppsala from the Swedish Research Council. J.B. is indebted to the Alexander von Humboldt foundation for financial support. The work of K.C. was supported by the Czech Science Foundation (Grant No. 15-08740Y). We thank Kristian Döbrich for stimulating discussions.

\section{Author contributions}

The high-order harmonics experiment was realized by R.C., B.F., M.T. and M.W., B.F., J.B. and R.C. recorded the data on gadolinium. Data analysis was done by B.F. and M.T The $a b$ initio calculations were performed by K.C. and P.M.O. atomistic spin-model 
calculations were performed by S.W., D.H. and U.N. All authors contributed to the discussion and preparation of the manuscript.

\section{Additional information}

Supplementary Information accompanies this paper at http://www.nature.com/ naturecommunications

Competing financial interests: The authors declare no competing financial interests.

Reprints and permission information is available online at http://npg.nature.com/ reprintsandpermissions/
How to cite this article: Frietsch, B. et al. Disparate ultrafast dynamics of itinerant and localized magnetic moments in gadolinium metal. Nat. Commun. 6:8262 doi: $10.1038 /$ ncomms9262 (2015).

cc (i) This work is licensed under a Creative Commons Attribution 4.0 International License. The images or other third party material in this article are included in the article's Creative Commons license, unless indicated otherwise in the credit line; if the material is not included under the Creative Commons license, users will need to obtain permission from the license holder to reproduce the material. To view a copy of this license, visit http://creativecommons.org/licenses/by/4.0/ 